\title{
The Effect of a 12-week Combined Exercise Intervention Program on Physical Performance and Gait Kinematics in Community-dwelling Elderly Women
}

\author{
Zhen-Bo $\mathrm{Cao}^{1)}$, ${\text { Akira } \mathrm{Maeda}^{2)} \text {, Norihiro Shima }}^{2)}$, Hiroshi Kurata ${ }^{2)}$ and Hidetsugu Nishizono ${ }^{3)}$ \\ 1) Graduate School of Physical Education, National Institute of Fitness and Sports in Kanoya \\ 2) Department of Sports Biomechanics and Applied Physiology, National Institute of Fitness and Sports in Kanoya \\ 3) Research Center for Training Science and Applied Physiology, National Institute of Fitness and \\ Sports in Kanoya
}

\begin{abstract}
This study aimed to determine if combined exercise intervention improves physical performance and gait joint-kinematics including the joint angle and dynamic range of motion (ROM) related to the risk of falling in communitydwelling elderly women. A 12-week combined exercise intervention program with extra emphasis on balance, muscle strength, and walking ability was designed to improve physical performance and gait. Twenty participants attended approximately two-hour exercise sessions twice weekly for 12 weeks. Participants underwent a physical performance battery, including static balance, sit and reach, whole body reaction time, $10 \mathrm{~m}$ obstacle walk, $10 \mathrm{~m}$ maximal walk, 30 -second chair stand, to determine a physical performance score, and received quantitative gait kinematics measurements at baseline and in 12 weeks. Significant lower extremity strength improvement $13.5 \%(p<.001)$ was observed, which was accompanied by significant decreases in time of the $10 \mathrm{~m}$ obstacle walk $(p<.05)$ and whole body reaction time $(p<.001)$ in this study. However, no significant differences were seen for static balance and flexibility from baseline. For gait kinematics, in the midswing phase, knee and hip joint angle changed toward flexion $(p<.01, \quad p<.05$, respectively). Ankle dynamic ROM significantly increased $(p<.05)$ following exercise intervention. The plantar flexion angle of the ankle in the toeoff phase was increased significantly $(p<.01)$. However, other gait parameters were not significantly different from baseline. These findings from the present investigation provide evidence of significant improvements in physical performance related to the risk factors of falling and safe gait strategy with a combined exercise intervention program in communitydwelling elderly women. The results suggest this exercise intervention could be an effective approach to ameliorate the risk factors for falls and to promote safer locomotion in elderly community-dwelling women. J Physiol Anthropol 26(3): 325-332, 2007 http://www.jstage.jst.go.jp/browse/jpa2
\end{abstract}

[DOI: 10.2114/jpa2.26.325]

Keywords: fall, physical performance, gait kinematics, exercise, elderly women

\section{Introduction}

Falls in older people are a major public health concern in terms of high morbidity, mortality, and enormous costs to health and social services. According to statistics of the Japanese Ministry of Health, Labour and Welfare in 2002, the elderly (over 65 yrs) who become bedridden owing to fracture by falling comprise $12.2 \%$ of the bedridden elderly, and is increasing. Furthermore, falling is the leading cause of injuryrelated deaths in elderly adults. For those aged 70-79 years, $27.7 \%$ of injury-related deaths are attributable to falling. This proportion increases to 46.4 and $64.8 \%$ for those aged $80-89$ years and 90-99 years, respectively (Rogers et al., 2003). Consequently, developing safe, effective, and feasible multidimensional exercise programs for preventing falls in elderly individuals is essential if we are to positively impact the health of the fastest growing population segment today.

Falls result from many factors, including both intrinsic and extrinsic. Intrinsic factors are internal to the individual. Increasing age, a history of falls, impaired balance, poor muscle strength, including ankle strength, slowed reaction time, and characteristics of gait changes are examples of intrinsic risk factors (Tinetti et al., 1988; Davis et al., 1999; Lord and Fitzpatrick, 2001; Schlicht et al., 2001). The most common gait changes related to the tendency to fall in older adults, in comparison to younger adults, are slower gait velocity with a shorter step length, shorter relative swing phase time, and less range of motion (ROM) at the hip, knee, and ankle joints (Judge et al., 1996; Kerrigan et al., 1998). The 
underlying joint kinetics associated with these kinematic reductions with age appear to be, primarily, lower plantar flexor torque and power at the ankle joint (Judge et al., 1996; Kerrigan et al., 1998), and, possibly, reduced torque at the knee and hip joints (Kerrigan et al., 1998).

Although some falls may have a single cause, most falls are believed to result from a combination of factors (Tinetti et al., 1988). Thus, it has been recommended that an exercise intervention program must include all exercise elements (strength, balance, flexibility, and endurance) to increase functional reserve, for long-term effective prevention of falls (Skelton and Beyer, 2003).

The effects of exercise on lower extremity muscle strength and these age-related gait changes have been reported for adults aged 60 years and older (Buchner et al., 1997; Judge et al., 1993; Lord et al., 1996). In addition, Lord et al. (1996) have demonstrated that training programs (aerobic, balance, and strengthening) have been proven successful at translating lower extremity muscle strength increases into improvement in gait in older adults. A limitation to previous work has been the selection of only one dependent measure, the gait velocity test, to assess the effectiveness of the exercise intervention. While the gait velocity test provides a global assessment of gait function, other dependent measures are needed to provide insight into mechanisms underpinning gait changes consequent to the exercise intervention. In addition, Savelberg and Meijer (2004) reported that the decline in muscle function that has been found to be associated with aging is joint angle dependent. Therefore, when investigating gait changes, it is necessary to consider changes at relevant joint angles. However, to our knowledge, few studies (DiBenedetto et al., 2005; Kerrigan et al., 2003) have investigated the effect of exercise intervention on the gait joint-kinematics including joint angle and dynamic ROM in elderly adults.

In this study, we examined the influence of a combined exercise intervention program on physical performance and gait kinematics in community-dwelling elderly women. Specifically, we tested the hypothesis that a combined exercise intervention program with extra emphasis on balance, muscle strength, and walking ability would improve physical performance (including static balance, sit and reach, whole body reaction time, $10 \mathrm{~m}$ obstacle walk, $10 \mathrm{~m}$ maximal walk, and 30-second chair stand performance) and gait jointkinematics including the joint angle and dynamic ROM related to the risk of falling in community-dwelling elderly women.

\section{Methods}

\section{Subjects}

Twenty healthy elderly women over 65 years old (age range, 65-79 yrs) were recruited to participate in a trial for preventing falls in a Health Promotion Center. All the subjects were independent in daily living activities and no walking aids were required. Subjects completed medical histories, exercise histories, and information on lifestyles by questionnaire and physical examination before participation in the study to ensure they were free from exclusion criteria. Exclusion criteria were the presence of severe cognitive impairment, symptomatic cardiovascular disease at moderate exertion level, the diagnosis of a stroke, Parkinson's disease, or other neurological disorder, peripheral neuropathy of the lower extremities and crippling arthritis. This study was approved by the Ethics Committee of the National Institute of Fitness and Sports in Kanoya.

\section{Exercise program}

The exercise program contained aerobic exercises, activities of balance, coordination exercises, strength training, and walking training. In particular, it included stretching, balance ball, step with body-bar exercises, mini-hurdle walking, zigzag footwork, pool walking, ordinary walking, and sit-and-stand up exercises. The exercisers participated in approximately twohour exercise sessions twice weekly for 12 weeks. The first 2 weeks of exercise was an acclimation period. The exercises were divided into five sections: a 5- to 10-minute warm-up period, a 15-minute stretching period, a 40-minute circuittraining period, a 30-minute balance ball exercise period or a 30-minute walking in pool period, and a 10-minute relaxation (cool down) period. One- to 5-minute rest periods were taken between exercise sections. Blood pressure was recorded as a safety measure only and was not recorded as an outcome before and after exercise sessions. The exercises were undertaken as group activities, with a major emphasis on social interaction and enjoyment.

\section{Outcome measures}

Physical performance measures in this study assessed several intrinsic parameters identified as risk factors for falling and future disability in older adults (Tinetti et al., 1988). Measurements were taken prior to beginning and completing the 12-week exercise program.

\section{Lower extremity muscle strength measurement}

Lower extremity muscle strength was determined via the 30Second Chair Stand Test (CS-30) which has been shown to be safe and effective in older adults without great expense (Jones et al., 1999). The test begins with the participant seated in the middle of a chair, back straight, and feet approximately shoulder width apart and flat on the floor. The arms are crossed and held against the chest. At the signal "go", the participant rises to a full standing position (body erect and straight) and then returns to the initial seated position. The participant is encouraged to complete as many full stands as possible within a 30 -sec time limit. The score is the total number of stands executed correctly within 30 seconds.

\section{Static balance}

Static balance was measured by recording how long subjects could remain standing on their self-reported dominant leg with their eyes open. Subjects were instructed to stand on the preferred foot while resting their hands at waist level and then raising the other foot approximately $5 \mathrm{~cm}$ off the floor with 
their eyes open. The test ends when the subject touches the free foot on the floor, removes the hands from the hips, moves the supporting foot from the original starting position, or hooks the free leg behind the support leg. The time until balance was lost (or maximum $120 \mathrm{~s}$ ) is recorded.

\section{Maximal walking speed}

Maximal walking speed was measured by timing subjects as they walked across a $10 \mathrm{~m}$ floor. The total length of the marked walkway course was $14 \mathrm{~m}$, allowing for $2 \mathrm{~m}$ acceleration and deceleration zones. Subjects performed two timed trials. Once testing was complete, the best time was recorded.

\section{$10 \mathrm{~m}$ obstacle walk}

Maximal walking speed was measured by timing subjects as they walked across a $10 \mathrm{~m}$ floor on which an obstacle was placed $(10 \mathrm{~cm}$ in height, $20 \mathrm{~cm}$ in thickness) at six places at intervals of $2 \mathrm{~m}$, including a start and goal point. Subjects performed two timed trials. Once testing was complete, the better time was recorded.

\section{Whole body reaction time}

Reaction time was measured by using a light signal as the stimulus and a jump from a force-plate as the response with a TKK-126b System. Each subject stood on a force mat and was asked to jump upright as quickly as possible in response to flashing. The record was computed from the average of 5 trials, not counting the maximum or minimum score for each subject. Sit and reach test

The sit and reach test was performed to evaluate the flexibility of the subject's trunk by a box type sit and reach apparatus. The subjects were instructed to sit with their feet positioned flat against the apparatus, with knees fully extended and feet together. As the experimenter pressed down on the knees to keep them extended, each subject was instructed to stretch forward slowly with hands and arms outstretched as far as possible toward or past the toes for at least $1 \mathrm{sec}$. Two trials were given; the better measurement reached was recorded.

\section{Gait analysis}

Subjects wearing sports shoes walked across a $10 \mathrm{~m}$ walkway, while sagittal kinematics data of the left lower extremity were recorded using a high-speed (125f/s) videotape camera system (HSV-500C3). Prior to videotaping, markers were placed on the left side of the subject at the following bony landmarks: the heel, the fifth metatarsal head, the lateral malleolus, the femoral epicondyle, the greater trochanter and the acromialis. Subjects were asked to walk on the walkway at a self-selected pace. The subjects were allowed to warm-up for a minimum of $5 \mathrm{~min}$. No fatigue effects were reported as a result of the warm-up for the elderly group. Subjects were asked to walk on the walkway at a self-selected pace after several practice trials. Kinematics data between $5 \mathrm{~m}$ and $8 \mathrm{~m}$ on the walkway were recorded for 1 gait cycles. The angles of hip, knee, and ankle at the phase of heel-contact (HC), mid-stance (MID-ST), toe-off (TO) and mid-swing (MID-SW) of the left foot were analyzed by software (WINanalyze Version 1.5). The $\mathrm{HC}$ phase was determined from the peak negative acceleration of the heel marker, and the TO phase was determined from the peak positive acceleration of the fifth metatarsal head marker (Mills and Barrett, 2001). MID-ST is defined as the phase in which the marker of the lateral malleolus is vertically below the marker of the greater trochanter in the stationary leg, and MID-SW is defined as the phase in which the marker of the lateral malleolus is vertically below the marker of the greater trochanter in the swing leg. The hip, knee, and ankle angles were measured relative to the vertical axis and thigh, thigh and shank, and shank and foot segments, respectively. Knee angle was computed by subtracting relative angle measurements from $180^{\circ}$. Dynamic ROM is defined as joint (Hip, Knee, and Ankle) angle range of motion during the gait cycle. The gait velocity was calculated for each trial by dividing the absolute stride length (defined as the distance traveled in two steps from $\mathrm{HC}$ to $\mathrm{HC}$ ) by the respective gait cycle duration (the stride time from $\mathrm{HC}$ to $\mathrm{HC}$ ). During the data collection period, subjects were asked to walk on the walkway at a self-selected pace after several practice trials.

\section{Statistical analysis}

Standard statistical methods were used to calculate mean and SD. To test changes in outcome measures between baseline and 12-week measurement data, a student's paired t-test was performed. Statistical significance was preset at the $p<.05$ level. All statistical analyses were undertaken using the Statistical Package for Social Sciences (Dr. SPSS for Windows).

\section{Results}

\section{Anthropometric characteristics}

There were no significant differences for anthropometric characteristics between baseline and 12-week measurement for height, body weight, percent body fat, and BMI (Table 1).

\section{Effect of exercise on physical performance}

No significant differences were seen between baseline and 12-week measurement for the time of the balance test and sit and reach (Table 2). The results for the performance and motor ability tests indicated that the time of the $10 \mathrm{~m}$ obstacle walk decreased $4.5 \%(p<.05)$ after exercise intervention. A similarly modest but not quite statistically significant improvement $3.8 \%(p=.10)$ in the time of the $10 \mathrm{~m}$ maximal walk was also observed after exercise intervention. The whole body reaction time decreased $6.4 \%(p<.05)$ significantly at the

Table 1 Anthropometric Variables at Baseline and 12-Week Measurement

\begin{tabular}{lrr}
\hline Variable & $\begin{array}{c}\text { Baseline } \\
(n=20)\end{array}$ & $\begin{array}{c}12 \text {-Week } \\
(n=20)\end{array}$ \\
\hline Height $(\mathrm{cm})$ & $148.97 \pm 7.29$ & $148.70 \pm 7.49$ \\
Weight $(\mathrm{kg})$ & $51.23 \pm 7.48$ & $50.69 \pm 7.27$ \\
Body Fat $(\%)$ & $27.73 \pm 4.33$ & $28.04 \pm 4.84$ \\
BMI $\left(\mathrm{kg} / \mathrm{m}^{2}\right)$ & $23.05 \pm 2.57$ & $22.91 \pm 2.65$ \\
\hline
\end{tabular}

Values represent mean $\pm \mathrm{SD}$ 
12-week measurement. It was found that the times of the CS30 test increased significantly, by $13.5 \%(p<.001)$, and this indicated a gain in muscle strength.

\section{Effect of exercise on gait}

Although stride length, cadence, and velocity tended to decrease, it was not a statistically significant decrease (Table 3 ). Figure 1 shows the hip joint angle, knee joint angle, and ankle joint angle at the phase of HC, MID-ST, TO, and MIDSW during a gait cycle. The hip joint angle of the HC, TO and MID-SW analysis phase during a gait cycle increased significantly ( $p<.001, p<.01, p<.05$, respectively). The knee joint flexion angle and the ankle joint plantar flexion angle at the phase of MID-SW during a gait cycle increased significantly $(p<.01, p<.05$, respectively). This means the foot clearance in the swing phase became greater after exercise intervention. The ankle joint plantar flexion angle at the phase of TO during a gait cycle increased significantly $(p<.01)$. No significant differences were noted between baseline and 12week measurements for dynamic ROM of the knee and hip joint. However, dynamic ROM of the ankle joint significantly increased $(p<.05)$ from 36.4 to 40.1 degrees after 12 weeks of combined exercise (Table 3 ).

\section{Discussion}

In this study, we have demonstrated that a 12-week combined exercise intervention program using inexpensive

Table 2 Changes in Physical Performance at Baseline and 12-Week Measurement

\begin{tabular}{lcc}
\hline \multicolumn{1}{c}{ Variable } & $\begin{array}{c}\text { Baseline } \\
(n=20)\end{array}$ & $\begin{array}{c}12 \text {-Week } \\
(n=20)\end{array}$ \\
\hline Static balance (sec) & $61.90 \pm 43.17$ & $65.78 \pm 45.36$ \\
Sit and reach (m) & $0.36 \pm 0.12$ & $0.36 \pm 0.09$ \\
Whole body reaction & $481.28 \pm 73.21$ & $450.50 \pm 66.24^{*}$ \\
$\quad$ time (msec) & & \\
10 m obstacle walk (sec) & $8.49 \pm 1.88$ & $8.11 \pm 1.64 *$ \\
10 m maximal walk (sec) & $5.28 \pm 1.00$ & $5.06 \pm 1.07$ \\
CS-30 test (times) & $14.80 \pm 4.03$ & $16.80 \pm 3.40 * * *$ \\
& & \\
\hline
\end{tabular}

Values represent mean $\pm \mathrm{SD}, * p<.05 ; * * * p<.001$

Table 3 Outcome Variables for Gait between Baseline and 12-Week Measurement

\begin{tabular}{lcc}
\hline \multicolumn{1}{c}{ Variable } & $\begin{array}{c}\text { Baseline } \\
(n=20)\end{array}$ & $\begin{array}{c}12 \text {-Week } \\
(n=20)\end{array}$ \\
\hline Velocity $(\mathrm{m} / \mathrm{s})$ & $1.37 \pm 0.15$ & $1.28 \pm 0.28$ \\
Stride length $(\mathrm{m})$ & $1.35 \pm 0.18$ & $1.28 \pm 0.19$ \\
Cadence (steps/s) & $122.45 \pm 11.06$ & $119.35 \pm 12.04$ \\
ROM of hip joint (deg) & $39.8 \pm 6.8$ & $41.5 \pm 7.3$ \\
ROM of knee joint (deg) & $69.7 \pm 5.9$ & $66.4 \pm 4.2$ \\
ROM of ankle joint (deg) & $36.4 \pm 5.9$ & $40.1 \pm 6.5^{*}$ \\
\hline
\end{tabular}

Values represent mean \pm SD ROM, range of motion during a gait cycle. $* p<.05$ equipment undertaken by community-dwelling elderly women can safely improve muscle strength. The strength gains could be associated with improvements in whole body reaction time, $10 \mathrm{~m}$ obstacle walk time, and gait joint-kinematics in community-dwelling elderly women. While long-term prospective studies of fall rates would be needed to confirm the potential benefits of combined exercise intervention, the findings from the present investigation provide evidence of significant improvements in physical performance related to the risk factors of falling and safe gait strategy after a combined exercise intervention program in communitydwelling elderly women.

\section{The effect of exercise intervention on physical performance}

Declines in static balance are also associated with falls (Tinetti et al., 1988), and static balance is a valuable predictor
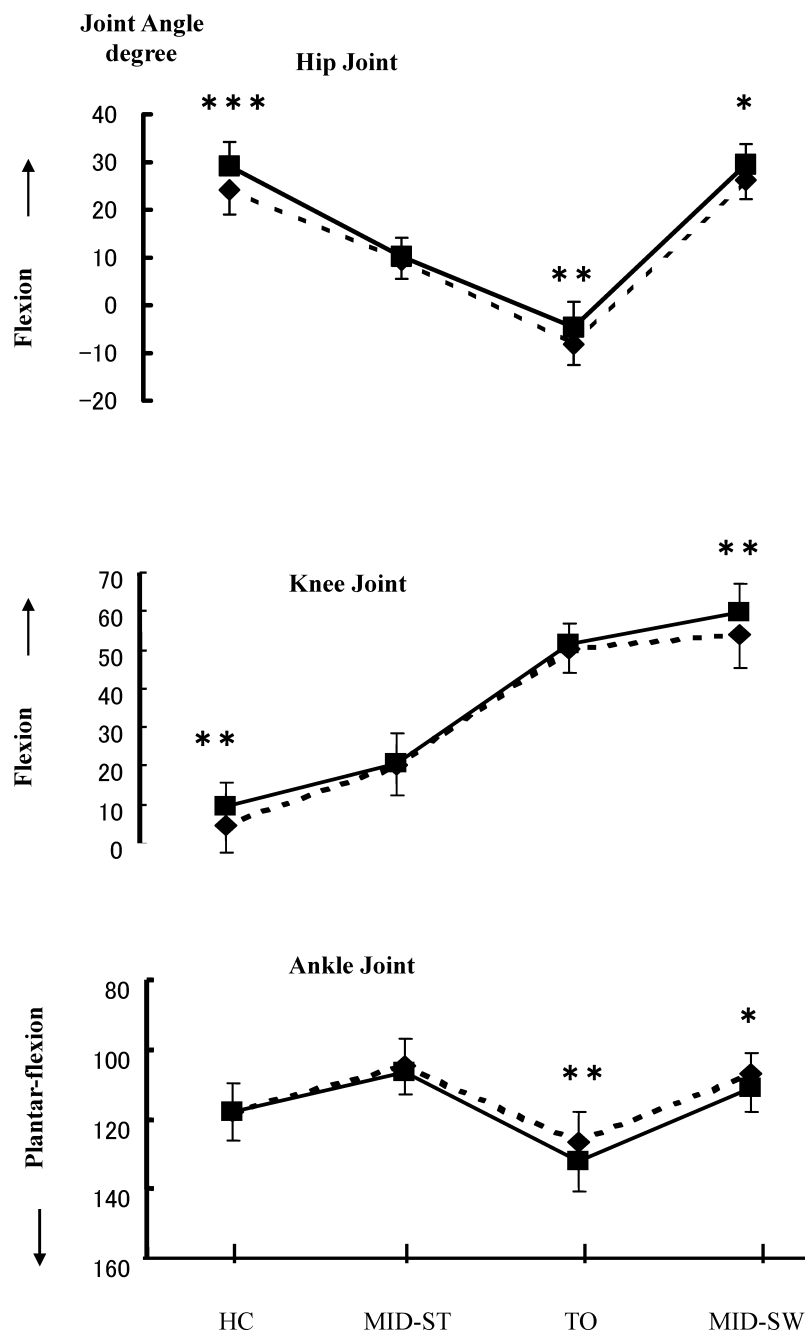

Fig. 1 The joint angle at the phase of heel-contact (HC), mid-stance (MID-ST), toe-off (TO) and mid-swing (MID-SW) during a gait cycle. Dotted lines represent the means of the baseline and solid lines represent the means of the 12 week measurement. ${ }^{*} p<.05 ; * * p<.01$; $* * * p<.001$ : significant different between baseline and 12 week measurements. 
for determining individuals who are at risk for falls (Rogers et al., 2003). Previous studies have investigated the effect of exercise intervention on static balance and have shown inconsistent results. Judge et al. (1993) reported that static balance in single stance improved $17 \%$ as a result of following combined training which included muscle resistance training of the leg (knee extension and leg press), walking exercise, and tai chi movements for six months, 3 times per week, for elderly women. Rikli and Edwards (1991) reported that static balance in a one-leg stance with eyes open was significantly improved following exercise for three years in older women. However, Engles et al. (1998) reported that no significant differences in static balance were seen following aerobic dancing (60 minutes per time, 3 times per week) in intensity (50-70\% HRmax) for elderly men and women. An improvement in leg muscle strength was also found in a study for improving the balance performance of elderly people (Islam et al., 2004; Englund et al., 2005). However, in this study, although leg muscle strength has improved, improvement of static balance was not seen. The reason seems to be that the subject's balanced capability baseline level was higher than in previous studies (Rikli and Edwards, 1991; Rooks et al., 1997; Englund et al., 2005). Differences in the age and previous activity levels of subjects may explain the difference of a subject's balanced capability baseline level. Moreover, the physical parameters associated with balanced capability is complex; it includes vision, perception, and proprioceptor systems. Reaction time is a physiological entity that has been studied extensively in the research literature and has been linked as a causal factor in the incidence of falls in the elderly population. Many strategies to improve reaction time dysfunction have shown inconsistent results. Whitehurst (1991) has reported that there was no effect of training on reaction time of elderly women, as a result of endurance training over 12 weeks (40 minutes per time, 3 times per week). Additionally, Panton et al. (1990) have also reported that there was no effect of training on the reaction time of elderly adults as a result of aerobic or resistance training over 26 weeks. However, Rooks et al. (1997) have reported that not only resistance training but also walking for 10 months has improved simple reaction time $(13 \%, 11 \%$, respectively). Our study extends the previous findings of Rikli and Edwards (1991), showing that combined exercise intervention for elderly women can improve reaction time. Differences in the mode and intensity of the exercises or in the contents of measurement in different studies may also explain the inconsistencies.

Previous exercise interventions to improve gait performance in older adults have been shown to be effective, within the limitation that most investigations have focused mainly on unobstructed gait performance (Schlicht et al., 2001; Puggaard, 2003). It has been shown that almost $50 \%$ of falls in community-dwelling older adults occur during destabilizing activities such as stepping over an obstacle and negotiating raised surfaces (Tinetti et al., 1995). Gait challenges such as stepping over an obstacle and negotiating a raised surface are commonly encountered during everyday activities and, compared with level walking, pose a greater threat to stability and place considerably increased demands on the muscular skeletal system (Patla and Rietdyk, 1993). Therefore, when investigating exercise interventions for preventing falls on gait performance, it is necessary to consider changes at obstacle negotiation walk performance. The significant decrease in the time of the $10 \mathrm{~m}$ obstacle walk after 12-week exercise intervention suggest an effective improvement in capacity to encountering difficult walk challenges in older women subsequent to the combined exercise intervention program in this study.

Research has consistently demonstrated that, given a stimulus of sufficient intensity, muscle strength increases in older adults (Rooks et al., 1997; Hurley, 1995; Lord et al., 1996). However, there are few exercise intervention studies in the literature examining the lower extremity muscle strength of subjects with the CS-30 test. Hruda et al. (2003) found that, using the CS-30 test, lower extremity muscle strength increased $66 \%$ using simple, progressive lower body resistance exercises. Islam et al. (2004) also reported that, using the CS30 test, lower extremity muscle strength increased 20\% using combined sensory and muscular exercise. These are higher improvement rates compared with the increase of $13.5 \%$ shown in this study. Differences in the mode and frequency of the exercise or in the age of subjects may also explain the difference of gain rates.

\section{The effect of exercise intervention on gait kinematics}

The findings of previous studies regarding the effects of exercise on gait velocity are inconsistent. For example, some researchers (Buchner et al., 1997; Sauvage et al., 1992; Brown and Holloszy, 1991) have reported little or no measurable benefit. In contrast, Judge et al. (1993) and Lord et al. (1996) reported that exercise groups achieved significant improvements in gait velocity. However, the effects of the intervention were heterogeneous in that those with initial slow walking speeds showed substantially improved walking speed at retest, while those with walking speeds that were normal to begin with showed little change. Additionally, Daley and Spinks (2000) have concluded that exercise influenced only slow walking speed in elderly people. In the present study, although there was a trend towards a decrease in gait velocity which is considerably faster than those of previous studies (Judge et al., 1993; Lord et al., 1996), it was not a statistically significant decrease. Differences in the baseline of gait velocity or in the contents of measurement in different studies may also explain the inconsistent finding. The reasons for the differences of gait velocity in those studies are unclear, but most likely reflect differences in both selection criteria and testing protocols.

However, few studies have focused on the gait jointkinematics presented here. It has been shown that the ankle of the elderly is more dorsi-flexed through the walking cycle than that of the young, and the dynamic ROM of the ankle joint is 
lower in the elderly compared to that in younger people (Winter, 1991). Judge and colleagues (1996) reported a reduced peak plantar flexion angle $(13 \pm 5$ degrees $)$ in the elderly compared with young adults (17 \pm 5 degrees). Prior studies have also reported reductions in ankle plantar flexion associated with reduced ankle power generation (Devita and Hortobagyi, 2000; Judge et al., 1996; Kerrigan et al., 1998). Increased hip extensor work is characteristic of elderly gait and has been proposed to compensate for a lack of trunk support in early stance (DeVita and Hortobagyi, 2000; Kerrigan et al., 1998), which is normally provided by the ankle plantar flexor (Neptune et al., 2001), while increased hip flexor work can potentially compensate for the decreased swing initiation (Judge et al., 1996) normally provided by the ankle plantar flexor. Additionally, it has been reported that fallers demonstrated only $37 \%$ of the knee extensor strength, and $10 \%$ of the ankle plantar flexor strength, exhibited by their nonfalling peers (Whipple et al., 1987). Therefore, it is considered that stronger propulsive forces during the last stage of the stance phase, in turn, resulted in improvement in the lower extremity muscle strength following a 12-week combined exercise program, because the results of the present study have shown an increase in the ankle joint angle at the phase of TO, and in the dynamic ROM in the ankle joint. These results extends the previous findings of exercise-related improvement in gait pattern in older adults (Lord et al., 1996), and shows that exercise intervention can be successful in translating lower extremity muscle strength increases into improvement in gait joint-kinematics in older adults.

Falls during walking are the primary cause of accidental injury in elderly individuals, and predominantly result from a slip or trip (Berg et al., 1997). Toe clearance has been considered to be a major responsibility of the swing leg dorsiflexors, and is quite sensitive to small angular change in the swing ankle, knee, and hip (Winter, 1992). During mid-swing, the toe drops to its minimum clearance, and the potential for a trip is greatest (Winter, 1992; 1991). Additionally, Chiba et al. (2005) have reported that the toe clearance of older fallers $(12.0 \mathrm{~mm})$ was significantly less than for older non-fallers $(15.2 \mathrm{~mm})$. The results of the present study have shown reduction in the ankle joint flexion (4.0 degrees) and increases in the knee (5.8 degrees) and hip (3.3 degrees) joint flexion at the phase of mid-swing. While our study did not address toe clearance directly, based on sensitivity calculations to determine the angular change (Winter, 1992), it is suggested that greater toe clearance during mid-swing resulted in improvement in lower extremity muscle strength following a 12-week combined exercise program. These results demonstrate the efficacy of exercise intervention in positively influencing fall-risk-related gait variables in community-dwelling older women.

This study has several limitations. First, the lack of controls weakens the internal validity of our major findings. Second, these results cannot be generalized to extremely weak or institutionalized elderly persons, because data were assessed in a group of relatively healthy community-dwelling elderly women of 65 years and older. Third, it is also acknowledged that a limitation of studies of this type is that subjects cannot be blinded to their "treatment" condition. Lastly, our study included only women participants to avoid potential sex effects. Consequently, the results may not apply to elderly men. Our results strongly suggest that combined exercise may be beneficial to elderly women for physical performance and for gait. Further study should involve larger sample sizes, controlled designs, and less healthy populations of older adults. Follow-up studies are also needed to determine if benefits for gait can be sustained over time for elderly populations.

In conclusion, a 12-week combined exercise intervention program with extra emphasis on balance, muscle strength, and walking ability resulted in an increase in significant improvements for physical performance, including whole body reaction, $10 \mathrm{~m}$ maximal walk, and lower extremity muscle strength, and improvement for gait joint-kinematics including the dynamic ROM of the ankle joint, ankle plantar flexion at the toe-off phase, and lower extremity joint angle at the phase of mid-swing during a gait cycle in community-dwelling elderly women. The results suggest that exercise intervention could be an effective approach to ameliorate the risk factors for falls and to promote safer locomotion in elderly communitydwelling women.

Acknowledgments Supported by a grant-in-aid (Research Program No. 14658011) from the Ministry of Education, Culture, Sports, Science and Technology of Japan.

\section{References}

Berg WP, Alessio HM, Mills EM, Tong C (1997) Circumstances and consequences of falls in independent community-dwelling older adults. Age Ageing 26: 261-268

Brown M, Holloszy JO (1991) Effects of a low intensity exercise program on selected physical performance characteristics of 60- to 71-year olds. Aging (Milano) 3: 129-139

Buchner DM, Cress, ME, de Lateur BJ, Esselman PC, Margherita AJ, Price R, Wagner EH (1997) The effect of strength and endurance training on gait, balance, fall risk, and health services use in community-living older adults. J Gerontol A Biol Sci Med Sci 52: M218-224

Chiba H, Ebihara S, Tomita N, Sasaki H, Butler JP (2005) Differential gait kinematics between fallers and non-fallers in community-dwelling elderly people. Geriatr Gerontol Int 5: $127-134$

Daley MJ, Spinks WL (2000) Exercise, mobility and aging. Sports Med 29: 1-12

Davis JW, Ross PD, Nevitt MC, Wasnich RD (1999) Risk factors for falls and for serious injuries on falling among older Japanese women in Hawaii. J Am Geriatr Soc 47: 792-798 
DeVita P, Hortobagyi T (2000) Age causes a redistribution of joint torques and powers during gait. J Appl Physiol 88: 1804-1811

DiBenedetto M, Innes KE, Taylor AG, Rodeheaver PF, Boxer JA, Wright HJ, Kerrigan DC (2005) Effect of a gentle Iyengar yoga program on gait. in the elderly: an exploratory study. Arch Phys Med Rehabil 86: 1830-1837

Engels HJ, Drouin J, Zhu W, Kazmierski JF (1998) Effects of low-impact, moderate-intensity exercise training with and without wrist weights on functional capacities and mood states in older adults. Gerontology 44: 239-244

Englund U, Littbrand H, Sondell A, Pettersson U, Bucht G (2005) A 1-year combined weight-bearing training program is beneficial for bone mineral density and neuromuscular function in older women. Osteoporos Int 16: 1117-1123

Hruda KV, Hicks AL, McCartney N (2003) Training for muscle power in older adults: effects on functional abilities. Can J Appl Physiol 28: 178-189

Hurley BF (1995) Age, gender, and muscular strength. J Gerontol A Biol Sci Med Sci 50: 41-44

Islam MM, Nasu E, Rogers ME, Koizumi D, Rogers NL, Takeshima N (2004) Effects of combined sensory and muscular training on balance in Japanese older adults. Prev Med 39: 1148-1155

Jones CJ, Rikli RE, Beam WC (1999) A 30-s chair-stand test as a measure of lower body strength in community-residing older adults. Res Q Exerc Sport 70: 113-119

Judge JO, Lindsey C, Underwood M, Winsemius D (1993) Balance improvements in older women: effects of exercise training. Phys Ther 73: 254-265

Judge JO, Underwood M, Gennosa T (1993) Exercise to improve gait velocity in older persons. Arch Phys Med Rehabil 74: 400-406

Judge JO, Davis RB 3rd, Ounpuu S (1996) Step length reductions in advanced age: the role of ankle and hip kinetics. J Gerontol A Biol Sci Med Sci 51: M303-312

Kerrigan DC, Todd MK, Della Croce U, Lipsitz LA, Collins JJ (1998) Biomechanical gait alterations independent of speed in the healthy elderly: evidence for specific limiting impairments. Arch Phys Med Rehabil 79: 317-322

Kerrigan DC, Xenopoulos-Oddsson A, Sullivan MJ, Lelas JJ, Riley PO (2003) Effect of a hip flexor-stretching program on gait in the elderly. Arch Phys Med Rehabil 84: 1-6

Lord SR, Lloyd DG, Nirui M, Raymond J, Williams P, Stewart RA (1996) The effect of exercise on gait pattern in older women: a randomized controlled trial. J Gerontl 51: 64-70

Lord SR, Fitzpatrick RC (2001) Choice stepping reaction time: a composite measure of falls risk in older people. J Gerontol A Biol Sci Med Sci 56: M627-632

Mills PM, Barrett RS (2001) Swing phase mechanics of healthy young and elderly men. Hum Mov Sci 20: 427-446

Neptune RR, Kautz SA, Zajac FE (2001) Contributions of the individual ankle plantar flexors to support, forward progression and swing initiation during walking. J Biomech 34: $1387-1398$
Panton LB, Graves JE, Pollock ML, Hagberg JM, Chen W (1990) Effect of aerobic and resistance training on fractionated reaction time and speed of movement. J Gerontol 45: M26-31

Patla A, Rietdyk S (1993) Visual control of limb trajectory during locomotion: effect of obstacle height and width. Gait Posture 1: 45-60

Puggaard L (2003) Effects of training on functional performance in 65, 75 and 85 year-old women: experiences deriving from community based studies in Odense, Denmark. Scand J Med Sci Sports 13: 70-76

Rikli RE, Edwards DJ (1991) Effects of a three-year exercise program on motor function and cognitive processing speed in older women. Res Q Exerc Sport 62: 61-67

Rogers ME, Rogers NL, Takeshima N, Islam MM (2003) Methods to assess and improve the physical parameters associated with fall risk in older adults. Prev Med 36: 255-264

Rooks DS, Kiel DP, Parsons C, Hayes WC (1997) Self-paced resistance training and walking exercise in communitydwelling older adults: effects on neuromotor performance. J Gerontol A Biol Sci Med Sci 52: M161-168

Sauvage LR Jr, Myklebust BM, Crow-Pan J, Novak S, Millington P, Hoffman MO, Hartz AJ, Rudman O (1992) A clinical trial of strengthening and aerobic exercise to improve gait and balance in elderly male nursing home residents. Am J Phys Med Rehabil 71: 333-342

Savelberg HH, Meijer K (2004) The effect of age and joint angle on the proportionality of extensor and flexor strength at the knee joint. J Gerontol A Biol Sci Med Sci 59: 11201128

Schlicht J, Camaione DN, Owen SV (2001) Effect of intense strength training on standing balance, walking speed, and sit-to-stand performance in older adults. J Gerontol A Biol Sci Med Sci 56: M281-286

Skelton DA, Beyer N (2003) Exercise and injury prevention in older people. Scand J Med Sci Sports 13: 77-85

Tinetti ME, Speechley M, Ginter SF (1988) Risk factors for falls among elderly persons living in the community. N Engl J Med 319: 1701-1707

Tinetti ME, Doucette J, Claus E, Marottoli R (1995) Risk factors for serious injury during falls by older persons in the community. J Am Geriatr Soc 43: 1214-1221

Whipple RH, Wolfson LI, Amerman PM (1987) The relationship of knee and ankle weakness to falls in nursing home residents: an isokinetic study. J Am Geriatr Soc 35: 13-20

Whitehurst M (1991) Reaction time unchanged in older women following aerobic training. Percept Mot Skills 72: 251-256

Winter DA (1992) Foot trajectory in human gait: a precise and multifactorial motor control task. Phys Ther 72: 45-53

Winter DA (1991) The biomechanics and motor control of human gait: normal, elderly and pathological. 2nd ed. University of Waterloo Press, Waterloo 
Received: October 27, 2006

Accepted: February 14, 2007

Correspondence to: Hidetsugu Nishizono, Director of Research Center for Training Science and Applied Physiology, National Institute of Fitness and Sports in Kanoya, 1 Shiromizu, Kanoya City, Kagoshima 891-2393, Japan

Phone: +81-994-46-5015

Fax: +81-994-46-5015

e-mail: nishizon@nifs-k.ac.jp 